\title{
Effects of attentional set and rhythmic complexity on attending
}

\author{
JAMES MOSHER KLEIN and MARI RIESS JONES \\ Ohio State University, Columbus, Ohio
}

\begin{abstract}
In a target detection task involving sustained attentional monitoring, rhythmic properties of tone sequences were found to affect detection performance (area under receiver-operating characteristic curves) and reaction times. Alternating tone frequencies (high, low) formed three different recurrent rhythms (binary, trinary, mixed) which varied in complexity. Attentional set was also manipulated so that participants attended either to tones of both frequencies (divided) or to only the higher of the two tones (selective). The most interesting finding involved an interaction between attentional set and rhythm, indicating that selective attending is enhanced by the most complex (mixed) rhythm, whereas divided attending tends to be best with the simplest rhythm (binary). Results are discussed in terms of a theory of dynamic attending, in which it is assumed that listeners actively use attending oscillators to direct attending.
\end{abstract}

In this article, we report the results of investigations on the influence of rhythm on attending. Traditionally, research on rhythm has focused on reproduction and/or perception of time intervals that make up a durational pattern. Two related questions have directed this research. The first, focused on determinants of rhythm reproduction, concerns why certain rhythms are harder to reproduce than others. Typically, tapping tasks require that listeners overtly produce or synchronize their responding with brief events (e.g., markers) separated by rhythmically patterned time intervals (see, e.g., Fraisse, 1963, 1984; Povel, 1981). Sometimes dual task settings are used in order to examine motor performance as a function of either central timekeepers or neuromotor oscillators (e.g., Kelso \& deGuzman, 1988; Rosenbaum, 1991; Summers, Rosenbaum, Burns, \& Ford, 1993; Wing \& Kristofferson, 1973a, 1973b). Time discrimination and time detection designs have been used to address the second question, concerning the role of rhythmic context on listeners' perception of various time relationships (e.g., Allan, 1979; Drake \& Botte, 1993; Halpern \& Darwin, 1982; Monahan \& Hirsh, 1990; Yee, Holleran, \&

This research is based on a thesis submitted by James M. Klein in partial fulfillment of the MA degree in psychology at Ohio State University. Following a brief illness, Jim Klein died on December 17, 1994; he completed revisions of the manuscript two weeks before his death. The authors gratefully acknowledge the contributions of Jim Klein's thesis committee members Neal F. Johnson and Richard Jagacinski, with special thanks to the latter for suggesting important auxiliary analyses. We thank Monica Weber, Eric Stallkamp, and Eric Riebel for their assistance in data collection. We are also grateful to Steve Handel, Stuart Klapp, Ed Large, Susan Holleran, John Platt, and Bruno Repp for comments on earlier versions of this manuscript. Finally, William Yee also deserves special thanks for assisting with manuscript preparation following Jim Klein's death. The research was supported in part, by a grant from the National Science Foundation, SBR-9412656, awarded to the second author. Requests for reprints should be directed to M. R. Jones, Department of Psychology, 137 Townshend Hall, Ohio State University, Columbus, OH 43210 (e-mail: mrjones@magnus.acs.ohio-state.edu).
Jones, 1994): Of interest here are psychophysical models of time discrimination (e.g., Allan, 1979; Killeen \& Weiss, 1987; Kristofferson, 1977).

In the present research, we relied on rhythmic manipulations in order to consider a different question raised by Jones and Boltz (1989), concerning the control of attending by event timing. When we listen to music (or speech), we typically attend to unfolding aspects of a pitch sequence (a melodic line). Jones and Boltz suggested that the rhythm traced out by such a melody affects how effectively we track its pitch pattern. We considered this question by using a task that does not systematically engage either motor control activities or time discrimination. Instead, it requires sustained attentional monitoring of tones that form temporally patterned sequences in order to detect alterations in their spectral structure. Rhythm as well as attentional set is manipulated in order to determine whether rhythmic structure can systematically influence attending to one (selective) or both (divided) of two different tone frequencies that constitute a given time pattern.

Central to understanding the influence of rhythmic structure on performance in any task is the problem of assessing structural complexity: How is rhythmic complexity defined and manipulated? Previous attacks on this problem come largely from rhythm production research. Using tapping tasks, both Deutsch (1983) and Povel (e.g., Povel \& Essens, 1985) generated influential hypotheses about structural determinants of memory codes for simple and complex rhythms. In a bimanual tapping task, Deutsch (1983) asked listeners to synchronously track (i.e., via tapping) various rhythms based on different pairs of isochronous trains (i.e., a high- and a low-frequency train). Performance was best when the rhythms were formed from two trains whose time intervals were harmonically related (e.g., 2:1, 4:2, and 3:1) and worst when they were related by complex ratios (e.g., $3: 5,5: 4$, etc.). She proposed that rhythmic complexity is based on the number of implied subdivisions; rhythms with 
complex time ratios yield more complex memory codes because they imply a greater number of temporal subdivisions per unit time (e.g., a 3:2 polyrhythm requires six subdivisions, whereas a $5: 4$ polyrhythm requires 20 ).

Povel assumes that rhythmic structure is coded via operations of a mental clock which prescribes a fixed grid of internal clock ticks as appropriate locations for pattern elements (e.g., tones). More complex codes occur with rhythms having more explicit deviations from ticks dictated by the grid of a best-fitting clock. Povel and Essens (1985) found that more difficult auditory rhythms contained more: (1) tones that did not coincide with prescribed "tick" locations; and more importantly, (2) silences that did coincide with "ticks" associated with the best clock.

Data spawned from such proposals have convincingly illustrated that rhythmic manipulations can affect memory and/or motor performance in tasks where listeners must produce or reproduce the time intervals between elements that outline a rhythm. However, neither the hypotheses themselves nor the tasks that they were designed to address speak directly to rhythmic effects on attending to elements, such as tones, that mark time intervals of a rhythm. Among other things, reliance on tapping tasks renders it difficult to tease apart rhythmic influences on motor coordination from those on attention. Indeed, as some contend, rhythmic complexity in motor tasks may be determined primarily by relative timing constraints on neuromotor oscillators, and not by either memory code complexity or structural constraints on attending (Kelso \& deGuzman, 1988; see also Rosenbaum, 1991). Current research has not resolved this issue, largely because production tasks inevitably involve memory and/or attention as well as overt motor responses.

Preliminary evidence suggests that rhythm may affect attending. Using a task that minimized motor involvement, Klapp et al. (1985) found that response times to terminal elements (e.g., a light or a tone) of different rhythms were significantly longer when the high- and low-frequency trains, which made up a rhythmic pattern, involved a complex (9:7) time ratio than when they involved a simple (1:1) one. If we assume that these people were monitoring all of the elements within these rhythmic sequences, then divided attending (i.e., attending to both frequency trains), like synchronous tapping, appears to be enhanced by simpler rhythms (see also Jones, Boltz, \& Kidd, 1982; Skelly, 1992).

In the present research, we pursued a more direct attack on the problem of temporal control of attending. We used a continuous long-term monitoring task in which listeners responded to elements (alternating high and low tones) that marked out one of three different rhythmic sequences: binary, trinary, and mixed (shown in Figure 1). Over each successive span of four tones (two high and two low), these three experimental rhythms were equivalent in terms of ordinal timing of high and low tones, average tone rate, and rates of high- and low-tone trains. Also all embedded a high-tone-frequency train of identical rhythmic structure (isochronous).

Figure 1 also shows several structural descriptions of these rhythms based on different approaches to rhythmic complexity. The conventional algorithms, advanced by
Deutsch (1983) and Povel (Povel \& Essens, 1985), can be adapted to the present task, in which both imply that attending performance should be most effective with the binary rhythm and least so with the mixed rhythm. That is, consider first the binary rhythm. It is based on a time ratio of $1: 1$ between periods that are marked out, respectively, by high and low tones (i.e., their respective interonset intervals, IOIs). Phase-shifted onsets of the low tones ( $\mathrm{L}$, L2) divide the respective time spans between successive high tones (H1, H2) into two equal parts; hence the rhythm's binary property. Deutsch's analysis dictates that fewer subdivisions are implied by this rhythm than by the trinary rhythm, which is based on a subdividing factor of $3: 1$; in the latter, low tones divide the same high-tone span into successive segments of $2 / 3,1 / 3$ and $1 / 3,2 / 3$ (based on three subdivisions per one high-tone span). The most complex rhythm, the mixed rhythm, has six subdivisions (each lasting $333 \mathrm{msec}$ ) for the same total time span. This rhythm, via a shift of one low tone, mixes subdivisions of binary and trinary rhythms; the first low tone, Ll, subdivides the first half of each cycle, as in the binary rhythm, whereas the second, L2, subdivides the second half following a $3: 2$ ratio, as in the trinary rhythm. In terms of absolute time properties, the mixed rhythm represents a very subtle timing shift in relation to the other two rhythms: only the onset of a single low tone is changed. A similar ordering of rhythmic complexity comes from the clock model of Povel and Essens (1985). The binary rhythm provides no deviations from the grid of the single best-fitting clock, and hence it is the simplest rhythm. Deviations, shown either as $\mathrm{d}$ for tones not occurring on a grid tick or as ds for ticks occurring during silences, occur in the other two rhythms; arguably, the mixed rhythm should be the most difficult of the three because it contains more of the troublesome deviations, ds. ${ }^{1}$

In addition to manipulating rhythmic structure, we also varied attentional set. In each of two attentional sets (selective, divided), listeners had to respond overtly when they heard a target within a designated subsequence of tones. In divided attending conditions, listeners had to listen for target tones randomly placed in both high-and low-frequency trains of a rhythmic sequence, whereas in selective attending conditions, listeners listened only for targets within the high-tone sequence (see also Jones, Jagacinski, Yee, Floyd, \& Klapp, 1995; Jones \& Yee, 1993; van Noorden, 1975). Within each attentional set condition, to-be-attended tones are termed relevant tones; targets constitute a subset of relevant tones that contain spectral alterations of their harmonic structure. Incentives and feedback (see Method section) reinforced attentional instructions.

Our primary reason for manipulating attentional set was to determine whether a listener's monitoring of different rhythms changes as a function of attending instructions. If instructions affect on-line performance, one interpretation of memory code models is that the effect of attentional set should be a general one that is not dependent on rhythmic complexity. In fact, there are independent reasons to expect that such a set manipulation will result in better performance with divided than with selective at- 

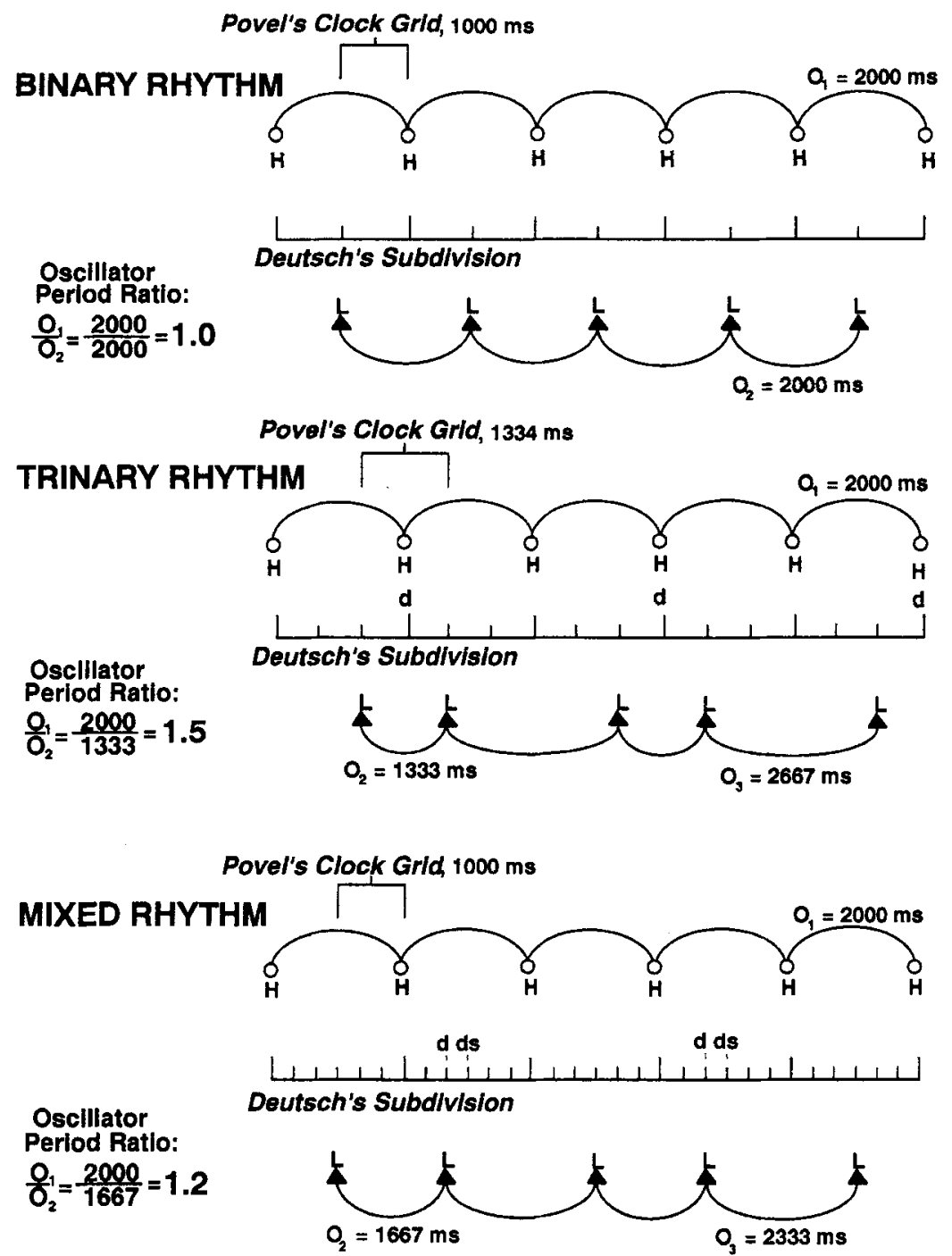

Figure 1. Rhythmic complexity analyses for binary, trinary, and mixed rhythms derived from hypotheses of Deutsch (1983), Povel (1981; Povel \& Essens, 1985), and Jones and Boltz (1989). In each rhythm, each high-tone onset defines a fixed time span of 2,000 msec, whereas low tones (filled triangles) vary to define a given rhythm. The symbols $d$ and ds refer to deviation types proposed by Povel and Essens (1985). (See text.)

tending, all other things being equal. ${ }^{2}$ That is, the divided attention instructions request listeners to "spread" (i.e., divide) attending over a rhythm's high- and low-frequency trains; hence they can encourage a reliance on relationships between successive, but different, tone frequencies. Indeed, these instructions emphasize this by telling listeners to integrate high and low tones to form a unified rhythm (Hirst, 1986; Jones \& Yee, 1993; Klapp, 1979). Taken together with recent findings from bimanual tapping tasks, which suggest that people are biased toward motor integration, it seems plausible that divided attending instructions will play into a natural tendency toward integration. The bias toward integration in motor tasks is evident in ostensible timing dependencies in bimanual coordination when one is tapping to a polyrhythm (Jagacinski, Marshburn, Klapp, \& Jones, 1988; Peters \& Schwartz, 1989;
Summers, 1990; Summers et al., 1993). In these contexts, subjects tend to interleave the two hands in a temporally interdependent manner; this bias for motor integration has recently been formalized in terms of a clock model of bimanual coordination (Summers et al., 1993).

Nevertheless, it is not clear whether the integration bias proposed for motor coordination, if present, is a purely motor (e.g., bimanual) phenomenon, or whether people have a more general tendency to attend to a succession of different elements (e.g., tones or hand gestures, etc.) as if they should be connected (i.e., related) in time. In brief, the tendency toward integration may not be strictly motor. If an integration bias obtains for attending as well, then, in a task requiring no synchronous tapping, instructions to divide attending should produce better monitoring than instructions to selectively attend, presumably because the 
former fit a natural inclination to hear the sequence as a unitary time pattern. In this paper, we refer to this as an attentional integrative bias hypothesis.

Other, more intriguing, interpretations raise the possibility of an interaction of rhythm with attentional set. An elaboration of the integrative bias hypothesis posits that rhythmic differences may arise because some time patterns fit more neatly with listeners' natural biases for integrative (divided) attending. This bias is most strongly reinforced by the binary rhythm, where time relationships most clearly (by the aforementioned complexity criteria) spell out a simpler, more unified, rhythm. Thus, one version of the integrative bias hypothesis implies that performance should be best in divided attending conditions with the binary rhythm; by the same token, because of a lack of compatibility with integration bias, performance should be worst in the selective-mixed condition. Another basis for predicting an interaction of rhythm with attentional set comes from adaptations of previously outlined rhythmic coding hypotheses. If listeners in selective attending conditions encode only the subset of time intervals relevant to a given attentional set, then one version of the coding approach implies that performance will differ as a function of rhythm in the divided attending conditions but not in the selective attending conditions. This is because, in the latter conditions, relevant tones (i.e., high tones) have the same time structure (isochronous) in all three rhythms.

Finally, rhythm and attention set may interact in yet another way if people use the rhythmic structure of the whole pattern to attend strategically. If the goal is to detect targets in both high- and low-frequency trains (i.e., in divided attending), then the time structure of binary rhythms, which facilitates integrating the periodicities that link successive tone frequencies, may best serve the function of targeting attending to all tones in these patterns. On the other hand, if the goal is to "tune out" one frequency (i.e., in selective attending), then the time structure of the mixed rhythm, which contains low tone irregularities, may best serve this goal.

This last interpretation is based on a theoretical framework outlined by Jones (1976; Jones \& Boltz, 1989). It assumes that attending is inherently temporal, involving entrainments of attending rhythms (i.e., attentional oscillators) to event periodicities marked within a rhythm. Unlike coding models, entrainment speaks to real-time control of attending and the generation of time-based expectations about forthcoming tones. Furthermore, in contrast to clock models, which emphasize the encoding of time interval invariances (e.g., grid intervals), attentional entrainments emphasize the direct response to ratio time invariances (see Jones \& Yee, 1993). To illustrate, assume that different attending rhythms (oscillators, $O_{i}$ ) can be excited by the recurrence of invariant time intervals among, respectively, high- and low-frequency tones; these attending oscillators can synchronize to invariant periods within each train. Ratio time relationships enter in to describe how attending rhythms relate to one another; connections among oscillators, via phase locking, depend on the nature and stability of the time ratio formed from their respective pe- riods, as summarized by an oscillator period ratio (OPR, Jones, 1976; Jones \& Boltz, 1989). ${ }^{3}$ Thus, a pattern containing simple and invariant time ratios, such as $1: 1,2: 1$, $3: 1$, is more likely to ensure invariant phase locking of two attending oscillators than is one with more complex ratios (Jones, 1976; see also, e.g., Glass \& Mackey, 1988).

According to this view, the ease of divided versus selective attending in a rhythmic context turns on how tightly the particular set of active attending oscillators, locked respectively to high- and low-tone periodicities, are themselves interconnected into a phase-locked arrangement. Divided attending conditions encourage use of tight oscillator connections, whereas selective attending conditions promote solo activity of the attending oscillator that tracks successive high tones ( $O_{1}$ in Figure 1$)$. When both set and rhythmic structure are compatible (dividedbinary; selective-mixed), relatively good performance should ensue; conversely, when an attentional set encourages reliance on an oscillator arrangement that is not readily achieved (divided-mixed, selective-binary), performance should be relatively poor.

Figure I illustrates this rationale. Periods of possible oscillators excited by each of the three experimental rhythms are shown as curved lines linking successive high or low tones. Two oscillators are shown for the binary rhythm and three each for trinary and mixed rhythms. ${ }^{4}$ The three rhythms also differ in induced OPRs. In the binary rhythm, both high and low tone frequencies consistently mark invariant periods of $2,000 \mathrm{msec}$; low-tone periods are phase shifted by $180^{\circ}$, expressing binary subdivisions. Attending oscillators, $\mathrm{O}_{1}$ and $\mathrm{O}_{2}$, are assumed to synchronize, respectively (with near-zero phase lags), to these time intervals. In addition, these oscillators should themselves readily connect with one another (via an invariant $180^{\circ}$ ) because they observe a simple (and invariant) $\mathrm{OPR}=\mathrm{O}_{2} / O_{1}=1.0$ Thus, divided attending should be good with a binary rhythm. By contrast, selective attending, which requires solo activity of $O_{1}$, should be relatively poor.

Trinary and mixed rhythms are predicted to promote less stable oscillator integrations in divided attending. Both excite more oscillators and afford more complex OPR values. In the trinary rhythm, $O_{1}$, with a 2,000-msec period, is locked to high-tone periods; however, the two low-tone periods $(1,333 \mathrm{msec}, 2,667 \mathrm{msec})$ excite oscillators $\mathrm{O}_{2}$ and $\mathrm{O}_{3}$ but only $\mathrm{O}_{2}$ permits attending to all low tones. In divided attending conditions, this means that listeners must learn to rely on $O_{1}$ and $O_{2}$ (with OPR of $3 / 2=1.5$ ). Finally, the mixed rhythm also engages three oscillators, but neither the $\mathrm{O}_{2}$ period $(1,667 \mathrm{msec})$ nor that of $\mathrm{O}_{3}$ $(2,333 \mathrm{msec})$ yields a simple OPR with $O_{1}$. Consequently, oscillator coordination in divided attending will be difficult, yielding poor performance. On the other hand, unlike binary and trinary rhythms, the mixed rhythm (e.g., an $\mathrm{O}_{1} / \mathrm{O}_{2}=1.2$ ) implies weak phase locking; this means that the mixed rhythm will facilitate separate reliance on individual oscillators. In other words, with the mixed rhythm, listeners should find it relatively hard to divide their attending, but relatively easy to selectively "tune out" the low tones and focus on the high tones (using $O_{1}$ ). 
In sum, in an attentional monitoring task, we vary rhythm and attentional set under the expectation that one or both of these variables will affect the speed and/or accuracy with which listeners detect alterations in tones that mark out various rhythmic patterns. Of particular interest is the interaction of attentional set with rhythm. From several perspectives, there is reason to anticipate that listeners will perform best in conditions in which they must divide their attention over tones that trace out a binary rhythm. However, with respect to selective attending, several different predictions are possible, including one associated with a dynamic attending approach which implies that the mixed rhythm should facilitate selective attending. In order to assess these predictions, three experimental rhythms (binary, trinary, mixed), along with two control rhythms (fast binary, high tone only), were used to examine divided and selective attending.

\section{METHOD}

\section{Design}

The full experimental design was a $2 \times 3 \times 2 \times 2$ mixed factorial. Three between-subjects factors were attentional set (selective, divided), rhythm (binary, trinary, mixed), and target counterbalance order $(1,2)$. The single within-subjects variable was target difficulty (hard, easy). A reduced design crossed the two levels of attentional set with three levels of rhythm for a single (hard) difficulty level. In addition, in supplementary analyses, two control conditions were used to assess selective attending with, respectively, a fast-binary rhythm and a high-tone-only rhythm.

\section{Participants}

A total of 192 participants, all with reportedly normal hearing, volunteered to participate in the study in return for credit in an introductory psychology course. Twelve were randomly assigned to each of the 12 between-subjects experimental conditions in the full design; in addition, 12 participants were randomly assigned to each of the two counterbalance orders for each of the two control conditions (fast binary; high tone only).

\section{Apparatus \\ All stimulus patterns were constructed and generated with the MIDILAB software system (Todd, Boltz, \& Jones, 1989). During each experimental session, patterns were presented on line by a Yamaha TX81Z tone generator controlled by an IBM AT computer with a Roland MPU-401 MIDI interface unit. Sequences of tones were amplified by a Kenwood KR-A4010 receiver and played over $\mathrm{AKG} \mathrm{K240}$ headphones at a comfortable listening level. A tone gen- erator voice having a timbre resembling an actual flute (termed Pan Floot) was used to generate all tones.}

\section{Stimulus Materials and Conditions}

The basic stimulus was a 4-sec pattern consisting of two high and two low tones, with each tone lasting $300 \mathrm{msec}$. A trial consisted of one presentation of this stimulus pattern (see Figure 1). All patterns were presented continuously in blocks of 30 unbroken trials; a trial set consisted of three blocks of trials (i.e., a total of 90 trials). Finally, an experimental session consisted of seven trial sets (i.e., a total of 630 trials).

\section{Stimuli}

The basic tone pattern consisted of rhythmically alternating low (L) and high $(\mathrm{H})$ frequencies. The low tone was based on a fundamental frequency of $\mathrm{C}_{4}$ (middle $\mathrm{C}, 261.6 \mathrm{~Hz}$ ); the higher tone was one octave above $\left(\mathrm{C}_{5}, 523.3 \mathrm{~Hz}\right)$. They were combined to form three experimental rhythms: binary, trinary, and mixed, as in Figure 1. These rhythms can be described in terms of a 4,000-msec interval, comprising two equal time spans between high tones: $\mathrm{HI}-\mathrm{H} 2$, and $\mathrm{H} 1-\mathrm{H} 2$. Within this larger interval, placements of low tones (L 1, L 2) implied underlying subdivisions of each high-tone interval with time ratios of $2: 1$ (binary), $3: 1$ (trinary), and $3: 1$ plus $2: 1$ (mixed), respectively (note, however, that explicit tone placements may differ, as in, e.g., the trinary rhythm, which evokes a $3: 2$ polyrhythm; see Figure 1). Average tone density (one tone per second) and high-tone timing were equivalent in all three experimental rhythms.

Two control rhythms were constructed for use in the selective attending conditions: the fast-binary and the high-tone-only control sequences. In the fast-binary, the four tones all occurred within a total time span of $2,667 \mathrm{msec}$ (instead of $4,000 \mathrm{msec}$ ), with all IOIs equal to $667 \mathrm{msec}$. This rhythm permitted assessment of effects of the higher response rates to targets necessitated by divided attending conditions because it presented high-tone targets at a rate equal to the target rate (both low and high tones) of experimental rhythms in the divided attending condition. The high-tone-only condition involved only high tones separated by 2,000 -msec IOIs; thus the hightone pattern was identical to that embedded within the three experimental rhythms. This rhythm permitted assessment of compliance with selective attending instructions by listeners hearing experimental rhythms, because control listeners receiving the high-toneonly rhythm could not, perforce, listen to low tones.

Modified (i.e., target) tones occurred randomly in both high- and low-frequency trains; they consisted of two brief frequency bursts within the steady state portion of a tone, 90 and $200 \mathrm{msec}$ after its onset. In both cases, the bursts corresponded to the addition of a frequency five semitones below the fundamental frequency of the altered tone (i.e., $\mathrm{G}_{3}$ or $196.0 \mathrm{~Hz}$ for $\mathrm{C}_{4} ; \mathrm{G}_{4}$ or $392.0 \mathrm{~Hz}$ for $\mathrm{C}_{5}$ ). Phenomenally, these spectral alterations sounded like "blips." Noticeability of blips was varied by adjusting both duration and amplitude of these added frequencies to create two different target difficulty levels (easy, hard) respectively for high- and low-tone trains. Equal difficulty levels for the two trains were determined on the basis of pilot work using judgments of four independent expert listeners. Unaltered high and low tones had amplitude levels of 71.7 and $70.9 \mathrm{~dB}$ SPL, respectively; these measurements were not changed appreciatively with addition of transient spectral components in that easy and hard $\mathrm{C}_{5}$ target tones were approximately 71.8 and $72.7 \mathrm{~dB}$ SPL; easy and hard $\mathrm{C}_{4}$ target tones were approximately 70.8 and $71.4 \mathrm{~dB}$ SPL, respectively. (Note: these measurements are necessarily approximations, given the transient nature of spectral alterations.) With low tones $\left(\mathrm{C}_{4}\right)$, all spectral bursts lasted $10 \mathrm{msec}$; with high tones $\left(\mathrm{C}_{5}\right)$, they lasted $5 \mathrm{msec}$.

On each trial, one of the four tones within the recurring stimulus pattern was spectrally altered; exceptions were the first trial and five other (catch) trials within each block, in which no altered tones occurred. The arrangement of both altered tones and of catch trials was randomized, although altered tones never occurred in the same temporal position in immediately successive trials. All conditions contained the same total number of altered tones, with the exception of the high-tone-only control, which contained half as many total tones (hence half as many altered tones). The number of functional targets (i.e., relevant altered tones) necessarily differed with attentional set. Given that a trial block lasted $2 \mathrm{~min}$, the percentage and rates of target tones were, respectively, as follows: In divided attention conditions, $20 \%$ were targets $(10 \%$ high-tone targets plus $10 \%$ low-tone targets), with a rate of 12 targets per minute, and in selective attention conditions, $10 \%$ were targets (altered high tones only), with a rate of 6 targets per minute. In the fast-binary control condition, also a selective attending condition, $13.33 \%$ of the tones were presented as targets at a rate of 12 per minute; the high-tone-only control condition had $20 \%$ targets at 6 per minute. These rates take into account that within each trial block, six catch trials containing no targets were randomly interspersed to prevent listeners from gen- 
erating specific temporal expectations. Overall, $80 \%$ of the tones were not altered.

In summary, five different rhythms were designed to be presented continuously over 630 trials. Three were experimental rhythms (binary, trinary, mixed), and two were control rhythms (fast-binary, high-tone-only). Experimental rhythms comprised alternating high and low tones having the same (isochronous) high-tone time sequence, and differing only with respect to timing of one low tone. Spectrally altered versions of high and low tones occurred randomly throughout the trials to serve as potential targets (except in the hightone-only control rhythm). Actual targets were defined as those spectrally altered tones relevant to a given attentional set; that is, the selective attending set rendered high tones relevant while the divided attending set rendered both high and low tones relevant. Control rhythms were designed to assess consequences of attentional set manipulations, such as target rate effects (fast-binary control) and compliance with instructions (high-tone-only control).

\section{Instructions and Procedure}

Attentional set was manipulated by instructions. In the divided attention condition, participants were told that both high and low tones were relevant and that they should respond by pressing a single response box button "as quickly as possible" in response to any change they detected in a tone, regardless of its pitch. In the selective attention condition, participants were told to attend only to the high tones and to respond to any alterations of these pitches; they were told to ignore all low-pitched tones. In addition, participants in both groups received instructions about accuracy and incentive levels. They were told that they could earn up to $\$ 2.00$ if they responded appropriately by adjusting their response criterion to each of the three different incentive levels they would encounter (low, medium, high).

After listening to the instruction tape, participants in all conditions heard four trials of a forthcoming stimulus pattern containing no altered tones. Next they heard seven trials of a pattern containing obviously altered low tones (except in the high-tone-only condition); this was followed by nine trials of a pattern with altered low tones identical to those in the experimental trials. A similar procedure was then followed with altered high tones.

Participants received seven sets of trial blocks. Each set comprised three trial blocks respectively involving different incentive levels (low, medium, high). Incentive level was manipulated to effect criterion shifts prerequisite to determining receiver-operating characteristic (ROC) measures of detection performance. Each of twelve participants within each of the between-subjects conditions was randomly assigned to one of three different orderings of incentive levels (low-medium-high, medium-high-low, high-low-medium) within a trial set ( $n=4$ per order/condition). Each of the three incentive levels obtained for a block of 30 trials, during which time participants received allotted points for their performance on each trial. Cards indicating the current incentive level and the number of points per hit at that incentive level were displayed for participants throughout each trial block. Points were automatically calculated by a computer program after each set of three blocks of trials, on the basis of the following payoff algorithm: (1) positive 10,20 , and 30 points per hit (i.e., detection of a target) in low-, medium-, and high-incentive conditions, respectively; and (2) negative 10 points for each false alarm and each miss (i.e., failure to detect a target) at all incentive levels. Correct rejections were not rewarded, resulting in three different payoff schedules. Participants received feedback on the number of points scored in each of the three incentive conditions, as well as their total number of points, after each of set of three trial blocks.

Participants were tested in groups of 1 to 4 . Each experimental session lasted approximately $1 \mathrm{~h}$ and $40 \mathrm{~min}$ (including a 5 -min rest break), after which each participant's total points were converted into a dollar value. The conversion rate ensured that all participants had the potential to earn the same maximum amount of money: For selective attention and high-tone-only groups, the rate was 0.04 cents per point; for divided attending groups, it was 0.02 cents per point; and for the fast-binary condition, it was 0.03 cents per point.

\section{Scoring on Dependent Measures}

In all conditions, responses which occurred between 300 and $967 \mathrm{msec}$ after the onset of a target tone were scored as hits. This range was chosen on the basis of pretesting with the group of participants used to equate difficulty levels. Their responses were never under $300 \mathrm{msec}$, and a $667-\mathrm{msec}$ response window is the maximum allowable, because some rhythms have IOIs of $667 \mathrm{msec}$. Responses in this range for unaltered tones were scored as false alarms. Hit and false alarm rates were calculated separately for high and low tones. Thus, no low-tone responses were included in any analysis of high tones. All responses falling outside this range were not scored.

In addition to response time, of interest is area under the ROC curve $(A)$, which ranges from 0.50 (chance) to 1.00 (perfect performance). An overall $A$ score was calculated for each participant (based on areas of trapezoids) by using an average of hit and false alarm rates over those determined for each of the seven trial sets (Green \& Swets, 1966; Macmillan \& Creelman, 1991). A separate ROC was determined for each participant at each difficulty level and tone frequency.

\section{RESULTS}

Analyses will be considered in two sections: Area Under ROC Curves $(A)$ and Reaction Times. In none of these analyses was counterbalance order a significant source of variance; accordingly, data were collapsed over this variable.

\section{Area Under ROC Curves $(A)$}

The $A$ score calculations are based on the assumption that listeners' response criteria are roughly equivalent within a given experimental condition and that they differ at the different incentive levels. An analysis of variance (ANOVA) indicated that listeners' false alarm rates (FARs), although generally low (means between $4 \%$ and $14 \%$ ), increased significantly over the three incentive levels $\left[F(2,276)=1,743.5, M S_{\mathrm{e}}=1.48, p<.00001\right]$. No other factors exerted a significant effect on FARs, nor did they provide significant interactions with incentive level. Error variance was small, not only suggesting homogeneity among listeners with respect to use of criteria, but probably also reflecting some range restrictions on FAR due to basement effects.

In this section, we present analyses of monitoring performance on both high- and low-frequency trains; ultimately, however, we focus on detection of high-tone targets for two reasons: (1) The (isochronous) high-tone time pattern is identical in all three rhythms, hence providing a fixed time structure against which to gauge global effects of binary, trinary, and mixed rhythms; and (2) in contrast to the low tones, the high-tone pattern is one to which listeners in both set conditions must respond, so that performance on these targets offers the opportunity to assess both set and rhythm as well as their interaction.

A preliminary analysis of high-tone performance indicated that although listeners performed better with easy than with hard targets, overall difficulty level did not modify effects of attentional set on rhythm. That is, listeners were best in detecting high-tone targets in the selective- 
mixed and divided-binary conditions, and this interaction of attentional set with rhythm was significant regardless of difficulty level $\left[F(2,138)=6.67, M S_{\mathrm{e}}=0.0212, p<\right.$ $.005]$. The three-way interaction of set with rhythm and difficulty level was not significant $(F=.64)$. Overall, difficulty level was a strong source of variance $[F(1,138)=$ $\left.320.81, M S_{\mathrm{e}}=0.0022, p<.0001\right]$, with listeners averaging .83 with easy targets and .73 with hard targets, overall. Target difficulty interacted with attentional set $[F(1,138)=$ $\left.7.13, M S_{\mathrm{e}}=.0022, p<.01\right]$; but, practically speaking, this was not a large interaction, and it was difficult to interpret. Finally, rhythm produced a significant overall effect $\left[F(2,138)=4.02, M S_{\mathrm{e}}=0.0212, p<.025\right]$, indicating that performance was best in the mixed rhythm; mean $A$ was .81 in the mixed rhythm, whereas it was .76 and .75 , overall, in binary and rhythm rhythms, respectively.

A parallel preliminary analysis was conducted on performance with low-tone targets. Necessarily, this analysis centers on performance of listeners receiving divided attending instructions. In these conditions, rhythm was not a significant source of variance. In fact, the only variable that produced a reliable effect on performance with lowtone targets was difficulty level $\left[F(1,69)=68.78, M S_{\mathrm{e}}=\right.$ $.0090, p<.0001]$. On the average, $A$ scores were lower for the more difficult low-tone targets.

These preliminary analyses indicated that responding was generally high with the easier targets, in some cases approaching ceiling effects. Accordingly, we focused our primary analysis on responding to the more difficult targets; here performance was closer to threshold levels and more sensitive to experimental manipulations. Figure 2 presents mean $A$ scores as a function of attentional set and rhythm for the hard targets; parallel data for the performance on the easy tones are presented in the Appendix.

In Figure 2, mean $A$ scores are plotted for hard targets that occurred in both the high- and low-tone trains. We consider first low-tone performance (i.e., in the divided attending conditions). An ANOVA indicated that rhythm had little effect on divided attending to low-tone targets.

We turn to performance on the high-tone targets. A full examination of effects of rhythm on performance with these tones includes the attentional set variable. Here we see that rhythm plays a major role, but that its degree of influence depends importantly on attentional set. An ANOVA on $A$ scores to the high-tone targets (excluding control conditions) confirms that, overall, rhythm significantly influenced performance with high-tone targets $[F(2,138)=$ $\left.3.62, M S_{\mathrm{e}}=.012, p<.05\right]$. Mean $A$ scores were 0.77 versus 0.71 for mixed versus binary plus trinary rhythms when averaged over the two attentional set conditions. As Figure 2 illustrates, although the mixed rhythm produced best overall performance, this was especially true in the selective attending condition. A reliable interaction of attentional set with rhythm persists in listeners' responses to these harder high-tone targets $\left[F(2,138)=6.51, M S_{\mathrm{e}}=\right.$ $.012, p<.005]$. In the selective attention condition, the average $A$ score for the mixed rhythm was 0.81 , roughly 0.10 units above those for the groups selectively attending to the other two experimental rhythms. In this analysis, the main effect of rhythm and its interaction with set provided the only two significant influences on high-tone performance. The lack of a main effect for attentional set is not consistent with a simple integrative bias hypothesis, in which divided attention is expected to be favored.

Two questions direct our analysis of the interaction of attentional set with rhythm. First, does rhythm differentially affect attending performance in selective versus divided attending conditions, and if so, how? Second, does attentional set significantly modify a listener's use of rhythmic structure? Using the Dunn test, we addressed these questions using five nonorthogonal planned pairwise comparisons $(C=5)$ over the six experimental conditions means; familywise error rate, $\alpha_{\mathrm{FW}}$, was fixed at .05 and $\alpha$ per contrast $=.01$ (Kirk, 1982, p. 106). Consider first differences due to rhythm. In the divided attending conditions, most theories predict that best performance should obtain with the binary rhythm; however, the anticipated superiority for this rhythm did not emerge in this set condition, at least for performance averaged over all trial blocks; the mean $A$ score of .74 was not statistically greater than the means for the other two rhythms in this condition (recall that a similar negative effect for rhythm obtained for performance with low tones in the divided attending condition). On the other hand, there was some evidence for the superiority of the binary rhythm early in the session; however, it was limited to comparisons between binary and trinary rhythm. In the first trial block of a session, listeners in the divided attending conditions were significantly poorer with the trinary than with binary and mixed rhythms combined (the latter two did not differ), yielding a Dunn $t$ test for this comparison of $\left[t_{\mathrm{D}}(138)=3.25\right]$. Nevertheless, the fact that the binary rhythm did not produce significantly higher $A$ scores than did the mixed rhythm in divided attending conditions is problematic for all theories.

In selective attending conditions, the picture changes: A strong effect of rhythm emerges. Over all trial blocks, people who were selectively monitoring high tones were significantly better when they received the mixed rhythm than when they received the binary rhythm $\left[t_{\mathrm{D}}(138)=\right.$ 3.80]. Indeed, listeners in the selective-mixed conditions, with an average $A$ score of .81, outperformed listeners in all other conditions, including those in the divided-binary.

The second question concerned whether attentional set modified a listener's use of rhythmic structure. There is evidence that this was the case, at least with respect to the mixed rhythm. Mean $A$ scores were significantly lower in the divided-mixed condition than in the selective-mixed condition $\left[t_{\mathrm{D}}(138)=3.28\right]$. Overall, attentional set effects are more directly addressed by refining the attentional set question as follows: Does performance on binary versus mixed rhythm reliably change as a function of attentional set? The relevant higher order comparison indicates that the answer is "yes" $\left[F(1,92)=12.06, M S_{\mathrm{e}}=.011, p<\right.$ $.001]$. When attending in a divided fashion, listeners were better with the binary than with the mixed rhythm, and this difference changed reliably with attentional set in such a way that for selective attending, listeners in the mixed rhythm performed distinctly better. 


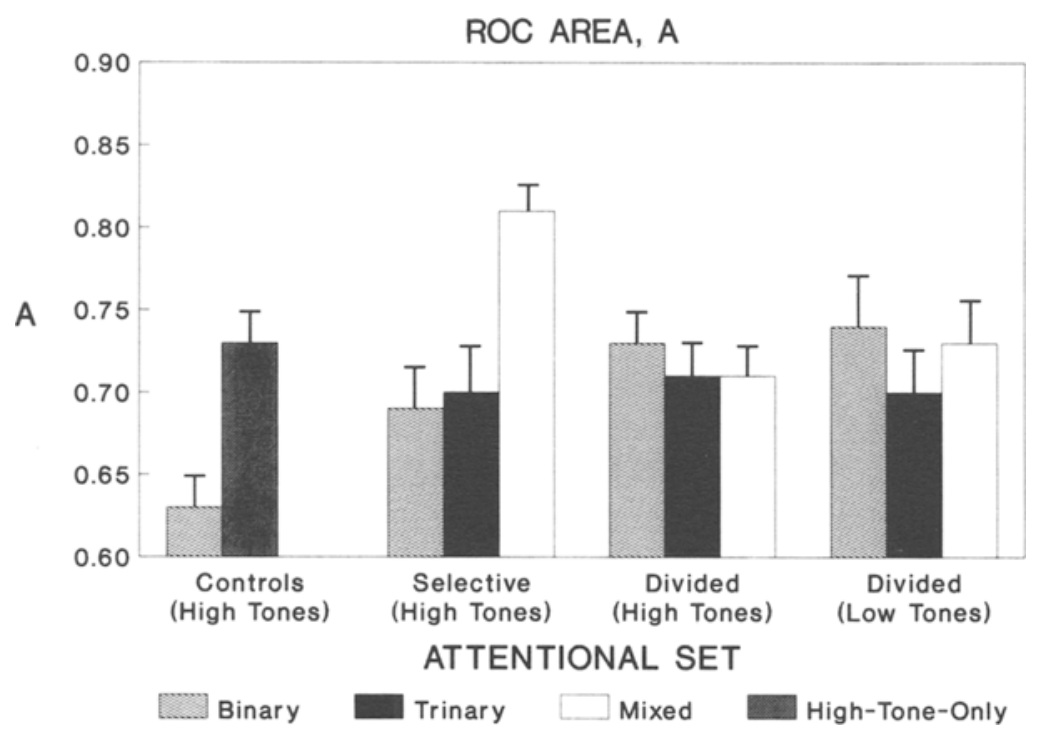

Figure 2. Mean $A$ scores, averaged over all seven trial sets, for hard targets in highand low-frequency trains for two control and six experimental conditions. The two control conditions are fast binary and high tone only; combinations of two attentional sets (selective, divided) with three experimental rhythms (binary, trinary, mixed) yield the six experimental conditions. Note that performance on low-tone targets is only possible in the three experimental conditions that involve a divided attending set.

Finally, we assessed the effectiveness of attentional set instructions. To determine whether selective attending listeners complied with instructions to ignore low tones, the role of the high-tone-only control group is critical because these listeners could only attend to the high tones. If listeners in other selective attending conditions performed at least as well as these control subjects, we may assume that they were following instructions. An overall ANOVA involving this control group along with the three selective attending groups confirmed that attentional set instructions were followed. A significant condition effect emerged $\left[F(3,92)=6.05, M S_{\mathrm{e}}=.0124, p<.001\right]$; however, a Dunn test $\left(\alpha_{\mathrm{FW}}=.05\right.$ for $C=3$ contrasts $)$ indicated that control listeners did not differ significantly from those in either the selective-binary or the selective-trinary conditions, and that they were reliably poorer than those in the selectivemixed conditions $\left[t_{\mathrm{D}}(92)=2.67\right]$. The latter outcome was unexpected; it suggests that the mere presence of to-beignored low tones may influence performance with the high tones. We defer discussion of thi point. For the present, these findings indicate that all listeners receiving selective attending instructions were following instructions. Converging evidence comes from response rates to the low tones, which were less than $1 \%$ in the selective attending conditions.

\section{Reaction Times}

The median reaction time of responses to correctly detected high- and low-tone targets (hits) was obtained for each of the seven trial sets at each difficulty level and incentive level. The mean of these was determined for each subject.
Preliminary analyses of reaction times to high tones included difficulty level as a variable; these yielded much the same outcome as did the analyses of $A$ scores-namely, a significant interaction of attentional set with rhythm $\left[F(2,138)=6.38, M S_{\mathrm{e}}=0.022, p<.025\right]$, which was not qualified by difficulty level. Also paralleling the $A$ score data, preliminary analyses of low-tone response times (in the divided attending condition) indicated modest, but statistically unreliable, effects of rhythm on speed of responding to these targets.

In order to pursue joint effects of set with rhythm, we continue to focus on high-tone performance with the more difficult targets. Means of median response times per trial set for the hard targets (high and low tones) are shown in Figure 3 as a function of attentional set and rhythm; parallel data for easy targets appear in the Appendix. Figure 3 indicates that performar ${ }^{\circ}$ is generally slowest in the selective-trinary condition and fastest in the selectivemixed condition. An ANOVA indicated that the only statistically significant influence on reaction times to these targets was the interaction of attentional set with rhythm $\left[F(2,138)=3.67, M S_{\mathrm{e}}=6,572, p<.05\right]$. Because of this, we first determ ned whether or not the predicted complex contrast that involved a change in performance in binary versus mixeu rhythms as a function of set was significant. It was $\left[F(1,92)=6.30, M S_{\mathrm{e}}=6,348.12, p<.025\right]$. People monitoring the binary rhythm were substantially slower than those monitoring the mixed rhythm with selective attendin $r$ instructions (i.e., by an average of $49 \mathrm{msec}$ ), and this difference reversed as a function of set, such that they became distinctly faster than listeners in the mixed rhythm with divided attending instructions (i.e., by $32 \mathrm{msec}$ ). 


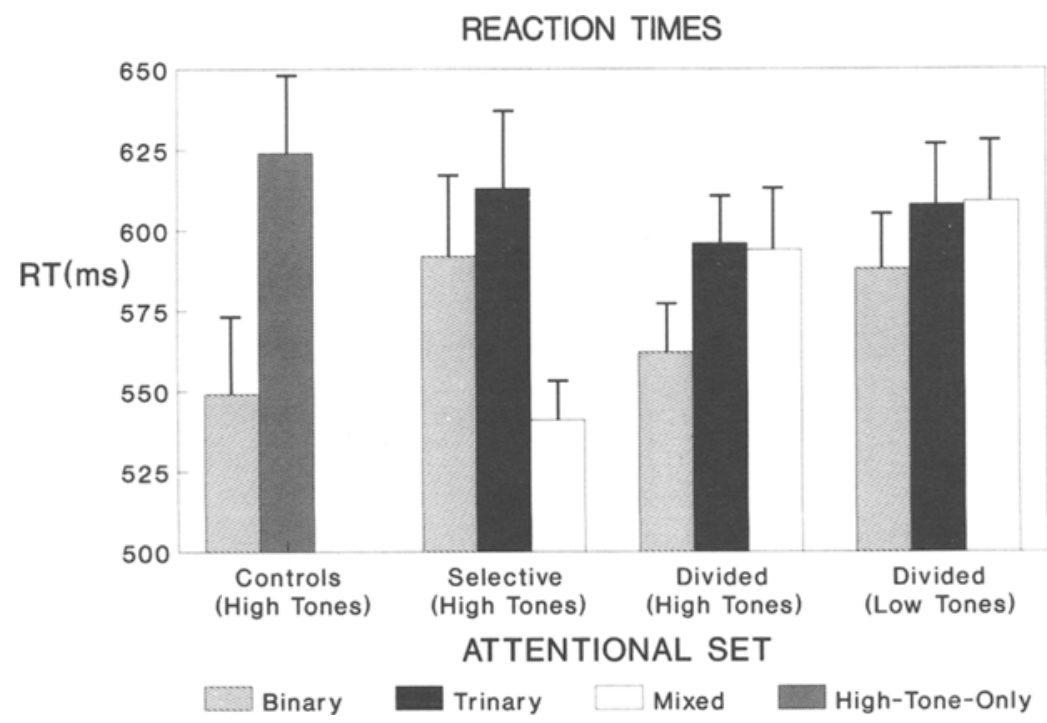

\begin{abstract}
Figure 3. Mean reaction time scores for hard targets in high- and low-frequency trains. These result from averaging median reaction times per trial set over all seven trial sets for each of two control and six experimental conditions. The two control conditions are fast binary and high tone only; combinations of two attentional sets (selective, divided) with three experimental rhythms (binary, trinary, mixed) yield the six experimental conditions. Note that performance on low-tone targets is only possible in the three experimental conditions that involve a divided attending set.
\end{abstract}

Using two $(C=2)$ Dunn pairwise comparisons, we found that response time differences due to rhythm were significant within the selective attending condition $\left[t_{\mathrm{D}}(138)=\right.$ 2.19] (see Kirk, 1982, p. 108), but not within the divided attending condition $\left(\alpha_{\mathrm{FW}}=.05\right.$ for one-tail Dunn comparisons with $\alpha=.025$ for each of the two predicted directional changes).

We also examined early session performance. The pattern of significant findings within the first trial block paralleled that of overall performance, but it was useful in revealing more clearly that when listeners must engage in divided attending, they initially have trouble doing so with the mixed rhythm. In fact, early in the session, the fastest responding occurred in the divided-binary and selectivemixed conditions. In these two conditions, listeners averaged 571 and $558 \mathrm{msec}$, respectively. By contrast, the conditions that produced slowest responding early in the session were the divided-mixed and selective-binary, with mean reaction times of 664 and $637 \mathrm{msec}$, respectively. Thus, in the first trial block, the reaction time measure presents a somewhat different picture of performance than did the $A$ score analysis for this trial block, especially with regard to the divided-mixed condition. At least with respect to the high-tone targets, performance was reliably slower in the divided-mixed condition than in the selective-mixed condition $\left[t_{\mathrm{D}}(138)=3.70\right]$. In fact, listeners in the divided-mixed condition were significantly slower on these high-tone targets than were listeners in the dividedbinary condition $\left[t_{\mathrm{D}}(138)=3.25\right.$; Dunn with $\alpha_{\mathrm{FW}}=.05$, $C=5]$. Finally, with respect to responding to low-tone targets early in the session, a similar pattern emerged in which responding was quicker in the binary than in the mixed rhythm in the divided attending condition. However, as with other analyses of performance with low-tone targets, these differences were not statistically significant.

Listeners in the high-tone-only control again performed comparably to listeners in selective attending conditions. In particular, in terms of response time, these listeners did not differ significantly from listeners in the selectivebinary and the selective-trinary conditions. The new information provided by this analysis is the discovery that, as shown in Figure 3, control listeners were reliably slower than listeners in the selective-mixed condition. This finding accounts for a significant effect of condition that emerged when performance of control listeners was assessed along with that of other selective attending conditions in an omnibus ANOVA $\left[F(3,92)=3.82, M S_{\mathrm{e}}=8532.02, p<.025\right]$; follow-up analyses confirmed that the only comparison that proved reliable was that between the control condition and the selective-mixed condition $\left[t_{\mathrm{D}}(92)=3.10\right.$; for $C=3$, $\alpha_{\mathrm{FW}}=.01$ of the Dunn test]. These findings indicate that listeners in the selective-mixed conditions, as gauged by the performance of this control group, were responding extremely efficiently to targets in the high-frequency train. Taken together with the ROC data, they also suggest that: (1) High $A$ scores (e.g., as selective-mixed) do not result from speed-accuracy tradeoffs; and (2) selective attending instructions were followed.

\section{Fast-Binary Control}

It is possible that instructions to divide attention between two different frequency trains handicaps listeners by virtue of requiring them to overtly respond to targets that necessarily turn over at a faster rate than in the selective 
attending conditions. The main analysis of $A$ scores and response times do not provide support for this hypothesis, in that no main effects of attentional set were observed. Nevertheless, it remains possible that a greater response effort is required in divided attending conditions and that this depressed the detection rates, thereby eliminating a potential main effect favoring the divided attention set in that analysis. If so, we might expect relatively poor performance from listeners told to selectively attend, for example, to a binary rhythm in which high-tone targets occur at the same rate as high-plus low-tone targets in the divided attending condition. This was the reasoning behind including the fast-binary condition; in this condition, the required response rate was identical to that of all divided attending conditions. Required response rate refers to the number of overt responses required, per unit time, for one to detect all specified targets (while making no false alarms) under a given attentional set. If response effort is a primary factor in performance, we expect listeners in the fast-binary control to perform equivalently to those in the divided attention conditions (where required response rate is identical) and more poorly than those in selective attending conditions (where required response rate is lower).

Overall, the data are mixed on this issue. A comparison of performance among the four conditions with equivalent required response rate (fast-binary control, divided-binary, divided-trinary, divided-mixed) indicated a significant effect of condition $\left[F(3,92)=4.89, M S_{\mathrm{e}}=.0102, p<.005\right]$ for $A$ scores, but not for reaction times $[F(3,92)=1.96]$. Follow-up comparisons of $A$ scores indicated that the fastbinary control differed only from the divided-binary where it yielded significantly lower $A$ scores (.634 vs. .734) $\left[t_{\mathrm{D}}(1,92)=3.73 ; C=3\right.$ for $\alpha$ per contrast of $\left..01 / C\right]$. This finding suggests that when required response rate is equivalent in the binary rhythm, an advantage exists for the divided attending set, at least with respect to $A$ scores. The fast-binary control condition did produce somewhat lower $A$ scores than those found in the slower selectivebinary condition (.634 vs. .691 ), but neither this difference $\left[F(1,46)=3.13, M S_{\mathrm{e}}=.0124, p<.10\right]$ nor the comparable one with reaction times $[F(1,46)=1.91]$ was statistically significant.

In short, required response rate may have contributed to lowering performance levels of listeners in the divided attending conditions, perhaps reducing detection accuracy (but not speed). But its contribution is apparently a modest one. The difference between the fast-binary control condition, where attending is selective, and the divided-binary condition leaves open a possibility of a small handicap for listeners who attend in a divided fashion. We return to this issue in the discussion.

\section{DISCUSSION}

The most important finding in this investigation is that rhythm, by itself, exerts a substantial influence on both the accuracy and speed of target detection in an attention task. The monitoring task was designed to minimize motor involvement and, indeed, to separate the overt detection re- sponse from event rhythm by randomly scheduling target occurrences over time. In this context, detection performance was, nevertheless, markedly affected by the nature of the underlying rhythm which carried target elements. Contrary to expectations derived from memory code assessments, the rhythm predicted to be most complex (the mixed rhythm) proved to support performance that was at least as good as that observed for the rhythm predicted to be least complex (the binary rhythm).

Performance on the binary rhythm provided some surprises. By most accounts, this rhythm should have produced good performance especially in divided attending conditions. But, at least in overall performance, this was not the case with either high- or low-tone targets. In the divided attending conditions, although there was a tendency for the binary rhythm to produce better performance, reliable differences in favor of the binary rhythm were not present. In part this may be due to the very subtle timing differences among the three experimental rhythms that were, otherwise, equivalent. Using much more complex rhythms, Klapp et al. (1985) did find faster reaction times to final elements of a binary rhythm than to those terminating a complex rhythm. But this cannot be the whole answer in the present case, because early in the session there was a significant advantage for the binary: People were reliably faster in responding to high-tone targets in the binary rhythm than to ones in the mixed rhythm when they had to divide their attending. In fact, in the first trial set, listeners in the divided-mixed condition were $93 \mathrm{msec}$ slower than those in the divided-binary condition! These data are important, because they are consistent with the general theoretical expectations that the binary rhythm is somehow simpler than others and should offer advantages to listeners who must divide their attending. At the same time, they suggest that these advantages may be transitory and confined to response speed.

The way a rhythm exerts its influence on performance depends on the function of the task. It appears that people come to use a rhythm to strategically attend to relevant tones. Listeners told to selectively attend only to high tones responded most quickly and accurately in a rhythm that, by conventional accounts, should be quite difficultnamely, the mixed rhythm. Such findings reflect pure rhythmic context effects, because the three selective attending groups (binary, trinary, mixed) monitored identical hightone sequences. That is, if the primary determinant of performance with high-tone targets is the isochrony of the tobe-attended frequency train (i.e., the high-tone rhythm), performance should have been equivalent across these three selective attending groups and, indeed, comparable to performance with the high-tone-only control condition. It was not. In fact, in the selective attending conditions, where listeners were supposed to ignore low tones, even a single timing "deviation" (relative to a binary rhythm) of one of the (irrelevant) low tones affected responses to high tones. This implies that even in the selective attending condition, the relative timing of both high and low tones affects how listeners use a rhythm. Not only were listeners in the selective-mixed condition better than their 
counterparts in the divided-mixed condition, but they were better than listeners in other two selective attending conditions (binary, trinary). A final point that underscores the fact that somehow people rely on the whole rhythmic context to promote a particular attending strategy comes from the finding that the performance of listeners in the selective-mixed condition also differed reliably from that of listeners in the high-tone-only control condition, where no low tones were presented.

The interaction of rhythm with attentional set is at the heart of theoretical differences about the role of rhythm in the present task. In particular, hypotheses based on coding algorithms, integrative bias, and dynamic attending predict different patterns of findings as a function of rhythm in the selective attending conditions. Neither the coding approach, which predicts no differences due to rhythmic manipulations in selective attending conditions, nor a version of the integrative bias hypothesis, which predicts that the selective-mixed condition should perform most poorly, accounts for the superiority of the mixed rhythm in the selective attending conditions.

As such, these findings highlight drawbacks in adapting a memory code approach to explain rhythmic influences on attending. By their very nature, memory accounts, such as Deustch's or Povel's, address an after-the-fact mental representation of a dynamic pattern. They share with more recent approaches to rhythm perception (e.g., Parncutt, 1994) an emphasis on retrospective codings of sets of time intervals. As result, although they offer hypotheses about perception of time intervals and rhythmic complexity, these hypotheses are not sufficiently flexible to explain how listeners differentially use an unfolding rhythm to direct attending to spectral aspects of tones. That is, in order to detect a target in this task, people do not have to reproduce a set of time intervals; they must simply learn to attend "at the right times" as a rhythm unfolds. We suggest that listeners learn to "use" the pattern of time intervals to influence the timing of attentional pulses that determine expectations (see also Jones et al., 1982; Kidd, 1993; Kidd, Boltz, \& Jones, 1984; Ralston, 1992). The present data provide the strongest evidence to date for the systematic influence of rhythm on attending to tonal elements.

We also found little support for the integrative bias hypothesis elsewhere in the data. It predicts a general superiority for divided attending conditions, but we found that overall performance was somewhat better for selective attending conditions. One mitigating outcome that suggests caution with respect to this conclusion comes from performance of listeners in the fast-binary control condition. These listeners selectively attended to the binary rhythm and performed more poorly than listeners who divided their attention in the same rhythm, although both groups had an equivalent required response rate. These findings can be interpreted to mean that detection performance in divided attending conditions is artificially lowered, owing to response effort requirements. This factor may underlie the less than superior performance of listeners in the divided-binary conditions.
For several reasons, we think that effects due to overt responding requirements are not a major source of difficulty for listeners in the divided attending conditions. First, listeners in the fast-binary control condition (who selectively attended), although performing worse than listeners in the divided-binary condition, nonetheless did not differ in accuracy from other rhythm groups with the same response requirement. Nor were these control listeners reliably slower than any of the divided attending groups. This indicates that differences due to attentional set manipulations were modest when required response rate was equated. Furthermore, an alternative, and equally plausible, interpretation of this finding is that listeners in the fast-binary control condition had difficulty simply monitoring the much faster patterns.

The findings from this experiment do not unambiguously support any single theory, but they are most congruent with the dynamic attending hypothesis that attending oscillators are enlisted in strategic configurations to serve goals dictated by attentional instructions. The most direct support comes from the significant interaction of attentional set with rhythm and higher order contrasts which indicate that overall performance differences due to binary versus mixed rhythm reverse significantly as a function of set (selective vs. divided). The mixed rhythm, assumed to engage weakly connected oscillators, was correctly predicted to promote relatively good selective attending and relatively poor divided attending. The binary rhythm, assumed to engage strongly connected oscillators, was correctly predicted to promote relatively good divided attending and relatively poor selective attending. Nevertheless, the finding that the mixed rhythm, overall, does not produce significantly poorer performance than does the binary rhythm within the divided attending condition is as difficult for the dynamic attending account as it is for other views. However, some of the data reported from early session performance are illuminating in this regard. They indicated that people were reliably slower (and somewhat less accurate) in responding to the high-tone targets in the mixed rhythm than in the binary rhythm when dividing their attending (although parallel effects were not present in performance with low-tone targets in this condition). This implies that some advantage accrues to binary rhythms in the divided attending condition, but that it may be transitory.

The possibility that certain rhythmic differences may wash out with experience raises questions about the role of rhythm in "tuning" attending over trials. Early in the session, both trinary and mixed rhythms slowed listeners' responses to the high tones in divided attending conditions. However, people in these conditions improved as trials progressed so that their response times more closely approximated those in the divided-binary condition. Why? Given a goal of divided attending, we surmise that learning to use these rhythms involves identifying and connecting the attending oscillators that will maximize overall hit rates. In the trinary rhythm, this entails activating oscillators $O_{1}$ and $O_{2}$ rather than $O_{1}$ with $O_{3}$ because en- 
gagement of $O_{3}$ would decrease hit rate (see Figure 1). In fact, early reliance on $\mathrm{O}_{3}$ may account for the relatively low $A$ scores of listeners in the divided -trinary condition in the first trial set. In the mixed rhythm, neither $O_{2}$ nor $\mathrm{O}_{3}$, when linked to $\mathrm{O}_{1}$, guarantees efficient temporal targeting of attending; furthermore, in both cases, these oscillator pairings are unstable (e.g., the OPR is 1.2). This instability means that the underlying oscillator structure can revert to a more stable ratio of $O_{1} / O_{2}=1.0$, associated with the binary rhythm. In this case, listeners may come to rely on this simpler ratio to develop a "getting-by" strategy: Use a simpler $(1: 1)$ coordination of two oscillators, but with a correction that incorporates a syncopation. This would accommodate the one out-of-phase low tone in the mixed rhythm (Jones, 1990).

Two remaining features of the data that deserve comment involve the performance of listeners in the control conditions. In the high-tone-only control condition, listeners attending only to high tones were reasonably accurate but fairly slow in responding. These findings offer two surprises. First, with respect to detection accuracy, participants in the selective-mixed condition actually do better than listeners in the high-tone control condition in which no distracting (low) tones are present. It is not clear why the mere presence of to-be-ignored low tones in the former condition had this impact on responding to high tones. One explanation assumes that when instructions encourage attentional focusing on high tones (i.e., as in selective attending), the mere presence of to-be-ignored lower frequencies forces a narrowing (hence heightening) of the attentional focus, an effect observed elsewhere (e.g., Dowling, Lung, \& Herrbold, 1987). However, this does not account entirely for the present data, because, as already noted, parallel effects were not evident in the selectivebinary and the selective-trinary conditions. This finding is particularly intriguing, because it implies that not merely the presence but the relative timing of to-be-ignored tones has some potential for narrowing the focus of attention.

Second, with regard to response times, the high-toneonly control listeners not only responded more slowly than those in the selective-mixed condition, they performed more slowly than listeners in all other conditions. A speedaccuracy tradeoff is unlikely, because $A$ scores in the hightone-only condition do not differ significantly from those of other selective attending listeners (excluding those in the selective-mixed condition) where reaction times tended to be faster. Alternatively, pattern rate in the hightone control condition was necessarily slower than in other conditions; thus, it is possible that these control listeners were pacing their attending according to the periodicities within these slower patterns. Related effects of attentional pacing have been found with visual polyrhythms (Skelly, 1992). If average pattern rate does pace attending, as revealed in reaction times, this would also account for performance in the fast-binary control condition. In this condition, pattern rate is faster than in the other conditions and reaction times are also faster. However, we cannot entirely rule out a speed-accuracy tradeoff, because $A$ scores are lower than those in the divided-binary. Nevertheless, it is possible that reaction times reflect the operation of more than one determining factor: task difficulty, uncertainty, and pattern rate.

In sum, the picture that emerges from these findings is fairly straightforward. In long-term attentional monitoring, rhythmic properties of the to-be-attended sequence affect attending to target elements. Reliance on rhythmic structure is strategic in such a way that certain rhythms are more likely to advance the goals of selective attending, whereas others are more compatible with divided attending.

\section{REFERENCES}

Allan, L. G. (1979). The perception of time. Perception \& Psychophysics, 26, 340-354.

DEUTSCH, D. (1983). The generation of two isochronous sequences in parallel. Perception \& Psychophysics, 34, 331-337.

Dowling, W. J., Lung, K. M.-T., \& Herrbold, S. (1987). Aiming attention in pitch and time in the perception of interleaved melodies. Perception \& Psychophysics, 41, 642-656.

Drake, C., \& Botre, M.-C. (1993). Tempo sensitivity in auditory sequences: Evidence for a multiple-look model. Perception \& Psychophysics, 54, 277-286.

FRAISSE, P. (1963). The psychology of time. London: Eyre \& Spottiwoode. FRAISSE, P. (1984). Perception and the estimation of time. Annual Review of Psychology, 35, I-36.

GLASS, L., \& MACKEY, M. M. (1988). From clocks to chaos: The rhythms of life. Princeton, NJ: Princeton University Press.

Green, D., \& SWeTS, J. (1966). Signal detection theory and psychophysics. New York: Wiley.

Halpern, A. R., \& DaRWIN, C. J. (1982). Duration discrimination in a series of rhythmic events. Perception \& Psychophysics, 31, 86-89.

Hirst, W. (1986). The psychology of attention. In J. E. LeDoux \& W. Hirst (Eds.), Mind and brain: Dialogues in cognitive neuroscience (pp. 105-141). Cambridge: Cambridge University Press.

JAGACINSKI, R., MARSHBURN, E., KLAPP, S. T., \& JONES, M. R. (1988). Test of streamed vs. integrated structure in polyrhythmic tapping. Journal of Motor Behavior, 20, $416-442$

JONES, M. R. (1976). Time, our lost dimension: Toward a new theory of perception, attention, and memory. Psychological Review, 83, 323-355.

JONES, M. R. (1990). Learning and the development of expectancies: An interactionist approach. Psychomusicology, 9, 193-228.

JONES, M. R., \& BOLTZ, M. (1989). Dynamic attending and responses to time. Psychological Review, 96, 459-491.

JONES, M. R., BOLTZ, M., \& Kido, G. (1982). Controlled attending as a function of melodic and temporal context. Perception \& Psychophysics, 32, $211-218$

Jones, M. R., Jagacinski, R. J., Yee, W., Floyd, R. L., \& KlapP, S. T. (1995). Tests of attentional flexibility in listening to polyrhythmic patterns. Journal of Experimental Psychology: Human Perception \& Performance, 21, 293-307.

JONES, M. R., \& YEE, W. (1993). Attending to auditory events: The role of temporal organization. In S. McAdams \& E. Bigand (Eds.), Thinking about sound: The cognitive psychology of human audition (pp. 69112). Oxford: Oxford University Press, Clarendon Press.

Kelso, J. A. S., \& DEGuzman, G. C. (1988). Order in time: How the cooperation between the hands informs the design of the brain. In H. Haken (Ed.), Neural and synergetic computers (pp. 180-196). Berlin: Springer-Verlag.

KIDD, G. (1993). Temporally directed attention in the detection and discrimination of auditory pattern components [Abstract]. Journal of the Acoustical Society of America (Pt. 2), 2315.

KidD, G., Boltz, M., \& Jones, M. R. (1984). Some effects of rhythmic context on melody recognition. American Journal of Psychology, 97, 153-173.

KIL.LEEN, P. \& WeISS, N. (1987). Optimal timing and the Weber function. Psychological Review, 94, 455-468. 
KIRK, R. E. (1982). Experimental design (2nd ed.). Belmont, CA: Wadsworth.

KLAPP, S. T. (1979). Doing two things at once: The role of temporal compatibility. Memory \& Cognition, 7, 375-381.

KlapP, S. T., Hill, M. D., Tyler, J. G., Martin, Z. E., Jagacinski, R. J., \& JONES, M. R. (1985). On marching to two different drummers: Perceptual aspects of the difficulties. Journal of Experimental Psychology: Human Perception \& Performance, 11, 814-827.

Kristofferson, A. B. (1977). A real-time criterion theory of duration discrimination. Perception \& Psychophysics, 21, 105-117.

Macmillan, N. A., \& Creelman, C. (1991). Detection theory: A user's guide. New York: Cambridge Unjversity Press.

Monahan, C. B., \& Hirsh, I. J. (1990). Studies in auditory timing: 2. Rhythm patterns. Perception \& Psychophysics, 47, 227-242.

PARnCUTT, R. (1994). A perceptual model of pulse salience and metrical accent in musical rhythms. Music Perception, 11, 409-464.

Peters, M., \& Schwartz, S. (1989). Coordination of the two hands and effects of attentional manipulation in the production of a bimanual $2: 3$ polyrhythm. Australian Journal of Psychology, 41, 215-224.

POVEL, J. D. (1981). Internal representations of simple temporal patterns. Journal of Experimental Psychology: Human Perception \& Performance, 7, 3-18.

Povel, J. D., \& Essens, P. J. (1985). Perception of temporal patterns. Musical Perception, 2, 41 1-440.

RALSTON, J. (1992). Accents in music. Unpublished doctoral dissertation, Ohio State University, Columbus.

Rosenbaum, D. A. (1991). Human motor control. San Diego, CA: Academic Press.

SKELlY, J. (1992). Pacing visual attention. Unpublished doctoral dissertation, Ohio State University, Columbus.

Summers, J. J. (1990). Temporal constraints on concurrent task performance. In G. R. Hammond (Ed.), Cerebral control of speech and limb movements (pp. 661-680). Amsterdam: Elsevier, North-Holland.

Summers, J. J., Rosenbaum, D. A., Burns, B. D., \& Ford, S. K. (1993). Production of polyrhythms. Journal of Experimental Psychology: Human Perception \& Performance, 19, 416-428.

TodD, R. E., Boltz, M., \& Jones, M. R. (1989). The MIDILAB research system. Psychomusicology, 8, 643-657.

VAN NOORDEN, L. P. A. S. (1975). Temporal coherence in the perception of tone sequences. Unpublished doctoral dissertation, Eindhoven University of Technology, Eindhoven, The Netherlands.

Wing, A. M., \& Kristofferson, A. B. (1973a). Response delays and the timing of discrete motor responses. Perception \& Psychophysics, 14, 5-12.

Wing, A. M., \& Kristofferson, A. B. (1973b). The timing of interresponse intervals. Perception \& Psychophysics, 13, 455-460.

Yee, W., Holleran, S., \& Jones, M. R. (1994). Sensitivity to event timing in regular and irregular sequences: Influences of musical skill. Perception \& Psychophysics, 56, 461-471.

\section{NOTES}

1. In the Povel and Essens (1985) model, a best-fitting clock is induced on the basis of accumulated counterevidence for each of several grid intervals. Evidence against a given clock is weighted so that unscheduled silences contribute greater contrary evidence (ds) than do misplaced tones (d). The best-fitting clock is the one with the least accumulated counterevidence. We note that this model differs from an earlier, hierarchical, coding model suggested by Povel in 1981 , in which uniform subdivisions of 3 and 2 provided more efficient time codes. In the clock grid model, counterevidence for a polyrhythmlike pattern, such as the present trinary rhythm, which arises from an underlying $3: 1$ subdivision, will be slightly greater than that for a binary rhythm. Both models (i.e., those in Povel, 1981; Povel \& Essens, 1985) predict that the mixed rhythm is most complex.

2. "All other things being equal" in the present context includes the effort to respond at a given rate to targets. In divided attending, targets occur at a faster rate than they do in selective attending conditions.

3. An oscillator refers to a biological rhythm with a recurrent period that is modifiable (within limits) via entrainment. Jones (1976) denoted the period of an attentional oscillator by $\Delta T^{\prime}$, where "each perceptual rhythm is adjustable within small and finite limits, to a real world time period, $\Delta T_{n}$, that falls within the range: $\Delta T^{\prime}=\Delta T_{n}+e_{n}$, where $e_{n}$ is distributed $N\left(\mu_{e n}, \sigma_{e n}\right)$ " (p. 340). Consequently, oscillators are less rigid and more responsive to real-world periodicities than are clocks; nevertheless, they cannot change periods willy-nilly. Furthermore, oscillators are entrained at particular ratios. Jones describes the parameter $C_{f}$ as referring to ratio time invariants in patterns themselves. This ratio property of a rhythm, in turn, affords a basis of oscillator integration where the internal estimate is denoted by $C_{t}^{\prime}$. Here OPR corresponds to $C_{t}^{\prime}$.

4. The two-oscillator model shown here with an OPR of 1.00 is the general case in which two different oscillators with the same period are engaged, but phase shifted. This configuration may apply when high and low tones are distinctly separated in frequency, thus requiring separate oscillators (e.g., see Jones et al., 1995). Nevertheless, we do not rule out the case in which listeners, with learning, replace two such oscillators with a single oscillator having a period that is one half the period of the original oscillators.

\section{APPENDIX}

Table A1

Overall ROC Scores $(A)$ and Reaction Times to Easy Targets (in Milliseconds)

\begin{tabular}{llllll} 
& \multicolumn{2}{c}{$A$ Scores } & & \multicolumn{2}{c}{ Reaction Times } \\
\cline { 2 - 3 } \cline { 5 - 6 } Conditions & $M$ & $S E$ & & $S E$ \\
\hline Selective: High Tones & & & & \\
$\quad$ Binary & .79 & .030 & & 564 & 22 \\
$\quad$ Trinary & .77 & .029 & 605 & 21 \\
$\quad$ Mixed & .89 & .010 & 517 & 10 \\
Divided: High Tones & & & & \\
$\quad$ Binary & .85 & .018 & 543 & 14 \\
$\quad$ Trinary & .82 & .018 & 573 & 14 \\
$\quad$ Mixed & .82 & .020 & 574 & 17 \\
Controls: High Tones & & & & \\
$\quad$ Fast binary & .74 & .012 & 516 & 15 \\
$\quad$ High tone only & .87 & .018 & 596 & 21 \\
Divided: Low Tones & & & & \\
$\quad$ Binary & .88 & .026 & 529 & 14 \\
$\quad$ Trinary & .82 & .027 & 540 & 18 \\
$\quad$ Mixed & .88 & .015 & 527 & 17 \\
\hline
\end{tabular}

Note- $S E$ is the standard error of the mean per condition.

(Manuscript received August 15, 1994; revision accepted for publication May 10, 1995.) 